\title{
Visualization of Local Magnetic Moments Emerging from Impurities in Hund's Metal
} States of FeSe

\author{
Song, Sang Yong; Martiny, J. H. J.; Kreisel, A.; Andersen, B. M.; Seo, Jungpil
}

Published in:

Physical Review Letters

Link to article, DOI:

10.1103/physrevlett.124.117001

Publication date:

2020

Document Version

Publisher's PDF, also known as Version of record

Link back to DTU Orbit

Citation (APA):

Song, S. Y., Martiny, J. H. J., Kreisel, A., Andersen, B. M., \& Seo, J. (2020). Visualization of Local Magnetic Moments Emerging from Impurities in Hund's Metal States of FeSe. Physical Review Letters, 124(11), [117001]. https://doi.org/10.1103/physrevlett.124.117001

\section{General rights}

Copyright and moral rights for the publications made accessible in the public portal are retained by the authors and/or other copyright owners and it is a condition of accessing publications that users recognise and abide by the legal requirements associated with these rights.

- Users may download and print one copy of any publication from the public portal for the purpose of private study or research.

- You may not further distribute the material or use it for any profit-making activity or commercial gain

- You may freely distribute the URL identifying the publication in the public portal 


\title{
Visualization of Local Magnetic Moments Emerging from Impurities in Hund's Metal States of FeSe
}

\author{
Sang Yong Song $\odot,{ }^{1}$ J. H. J. Martiny $\odot,{ }^{2}$ A. Kreisel® ${ }^{3}$ B. M. Andersen $\odot,{ }^{4}$ and Jungpil Seo $\odot^{1, *}$ \\ ${ }^{1}$ Department of Emerging Materials Science, DGIST, 333 Techno-Jungang-daero, Hyeonpung-Eup, \\ Dalseong-Gun, Daegu 42988, Korea \\ ${ }^{2}$ Center for Nanostructured Graphene (CNG), Department of Physics, Technical University of Denmark, \\ DK-2800 Kongens Lyngby, Denmark \\ ${ }^{3}$ Institut für Theoretische Physik, Universität Leipzig, D-04103 Leipzig, Germany \\ ${ }^{4}$ Niels Bohr Institute, University of Copenhagen, Lyngbyvej 2, DK-2100 Copenhagen, Denmark
}

(Received 23 July 2019; revised manuscript received 30 September 2019; accepted 21 February 2020; published 17 March 2020)

\begin{abstract}
Understanding the origin of the magnetism of high temperature superconductors is crucial for establishing their unconventional pairing mechanism. Recently, theory predicts that $\mathrm{FeSe}$ is close to a magnetic quantum critical point, and thus weak perturbations such as impurities could induce local magnetic moments. To elucidate such quantum instability, we have employed scanning tunneling microscopy and spectroscopy. In particular, we have grown FeSe film on superconducting $\mathrm{Pb}(111)$ using molecular beam epitaxy and investigated magnetic excitation caused by impurities in the proximityinduced superconducting gap of FeSe. Our study provides deep insight into the origin of the magnetic ordering of FeSe by showing the way local magnetic moments develop in response to impurities near the magnetic quantum critical point.
\end{abstract}

DOI: 10.1103/PhysRevLett.124.117001

FeSe presents intriguing properties in terms of the interplay among the lattice, charge, and spin degree of freedom. Its nematic phase transition occurs at $T_{S}=90 \mathrm{~K}$, below which the $C_{4}$ lattice symmetry is reduced to $C_{2}$ symmetry [1-3]. Unlike other iron-based superconductors, however, the long-range magnetic ordering is absent in FeSe down to the superconducting transition temperature $T_{c}=8 \mathrm{~K}$ for bulk material, making the degree of freedom that drives the nematic order ambiguous among lattice, charge, and spin [3-10].

Although long-range magnetic ordering is absent in $\mathrm{FeSe}$, there are experiments that suggest the ground state of FeSe is close to the magnetic quantum phase transition point. The hydrostatic pressure of $\sim 1 \mathrm{GPa}$ induces static stripe antiferromagnetic (AFM) orders in FeSe which are typically observed in other iron-based superconductors [11-14]. There is also evidence of local magnetism in FeSe. For example, a muon spin resonance ( $\mu \mathrm{SR})$ study of $\mathrm{FeSe}_{0.85}$ measured an exponential decay of the muon polarization, which might hint at the presence of randomly oriented local magnetic moments [15]. The magnetostriction and susceptibility experiment shows strong in-plane anisotropy in FeSe, inferring the coupling of the local magnetic ordering and spin-orbit coupling [16]. Recently, a scanning tunneling microscopy (STM) study observed a signature of local spin fluctuations near the Fe defect in multilayer FeSe on $\mathrm{SrTiO}_{3}$ substrate [17]. Such magnetic instability suggests the possibility that the magnetism can be triggered by impurities in FeSe [18-20].
Despite intensive efforts in understanding the magnetism in FeSe, the direct observation of local magnetic moments emerging from impurities, which results from the quantum instability, has been challenging mostly owing to the lack of spatial magnetic resolution in the experiments. Here, we report a novel experimental approach to investigate the impurity-induced local magnetic moments in FeSe. Using molecular beam epitaxy, we grow FeSe film on $\mathrm{Pb}(111)$ substrate which is known to be an $s$-wave superconductor. We have observed a clear signature of the $s$-wave superconducting gap on the FeSe film, which is proximity induced from the $\mathrm{Pb}$. When magnetic moments are developed near impurities in FeSe, they respond to the $s$-wave superconductivity and provide exchange potentials to break Cooper pairs. This leads to strong bound states known as Yu-Shiba-Rusinov (YSR) excitation within superconducting gap [21-25]. Thanks to the extreme sensitivity of superconductivity to magnetism, the energy and spatial resolutions in our study for observing the local magnetic moments are unprecedented. All data are taken at $4.3 \mathrm{~K}$.

In the growth of $\mathrm{FeSe}$, we first grew a single layer (SL) of $\mathrm{PbSe}$ on $\mathrm{Pb}(111)$. Subsequently, we deposited $\mathrm{Fe}$ atoms on the $\mathrm{PbSe}$ at $490 \mathrm{~K}$, which resulted in the formation of FeSe islands on $\mathrm{Pb}(111)$ (Supplemental Material [26]). Figure 1(a) shows a typical STM image of the FeSe island grown on $\mathrm{Pb}(111)$. The FeSe island is surrounded by the $\mathrm{PbSe}$ layer and several bare $\mathrm{Pb}$ patches appeared during the growth. The hexagonal-shaped defects in the island and near the island are $\mathrm{Ar}$ gas bubbles trapped inside the $\mathrm{Pb}$ 


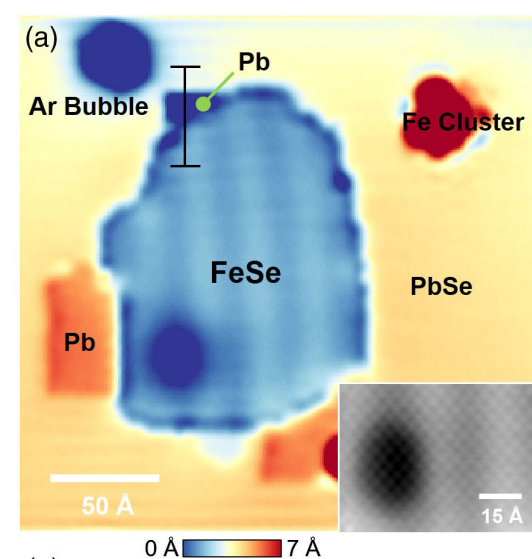

(e)

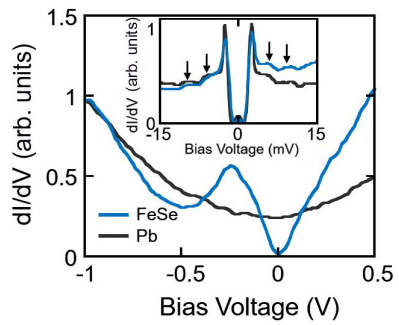

(b)

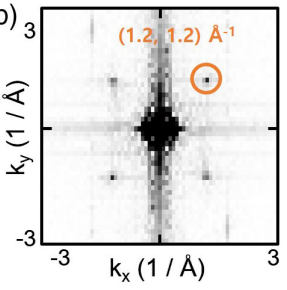

(c)
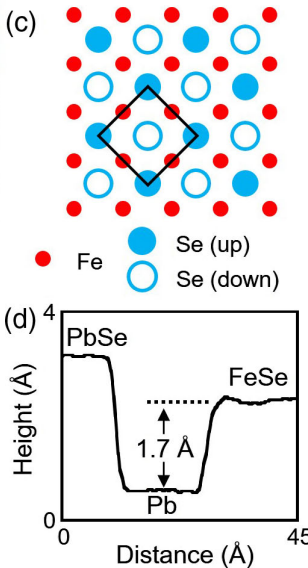

FIG. 1. (a) The topography of FeSe island grown on $\mathrm{Pb}(111)$. $V_{\text {bias }}=-0.1 \mathrm{~V}$ and $I=50 \mathrm{pA}$. The inset shows the atomically resolved image. (b) Fourier transform of the FeSe image. (c) Atomic model of the FeSe. The solid box represents the Se lattice in the top layer. (d) The height profile along the vertical line in (a). (e) The $d I / d V$ spectra measured in the FeSe island and bare $\mathrm{Pb}$. $V_{\text {bias }}=-1 \mathrm{~V}$ and $I=50 \mathrm{pA}$. Lock-in: frequency $f=$ $463.0 \mathrm{~Hz}$ and root-mean-square (rms) amplitude $V_{\text {rms }}=10 \mathrm{mV}$. The inset shows the spectra around the Fermi energy. $V_{\text {bias }}=$ $-15 \mathrm{mV}$ and $I=50 \mathrm{pA}$. Lock-in modulation: $V_{\mathrm{rms}}=0.3 \mathrm{mV}$. The arrows indicate the phonon peaks derived from the $\mathrm{Pb}$ superconductivity.

substrate [30]. The inset shows FeSe surface with atomic resolution. A rectangular lattice structure is clearly resolved and is distinguished from the crystal structure of $\mathrm{Pb}(111)$.

Figure 1(b) shows the Fourier transform of the topography of the FeSe. The lattice peaks are present at the position of $\left(k_{x}, k_{y}\right)=( \pm 1.2, \pm 1.2) \AA^{-1}$, whose numbers translate into the lattice constant of $3.7 \AA$. Figure 1(c) depicts the atomic model of FeSe forming trilayer (TL) structure. The blue-filled circles, the red-filled circles, and the blue-open circles represent the Se atoms in the top layer, the $\mathrm{Fe}$ atoms in the middle layer and the Se atoms in the bottom layer, respectively. As STM mostly measures the topmost Se atoms [31,32], the obtained lattice structure should conform to the Se lattice in the top layer [sold box in Fig. 1(c)]. For bulk FeSe, the Se lattice constant is known to be $\sim 3.75 \AA$ [33-35], which agrees well with the measured value.

The apparent height of FeSe islands with respect to the $\mathrm{Pb}$ substrate is found to be $\sim 1.7 \AA$ [Fig. 1(d)]. This is smaller than the $1 \mathrm{TL}$ of bulk FeSe $(\sim 5.33 \AA)$, indicating that most of the FeSe is embedded inside $\mathrm{Pb}$. Similar

growth has been reported when FeSe film is grown on soft substrates [36-38]. The lattice modeling of FeSe and $\mathrm{Pb}$ estimates the thickness of our FeSe is 3 TL [26]. A moiré pattern found in the FeSe [Fig. 1(a)], which is due to the lattice mismatch between FeSe and $\mathrm{Pb}(111)$, confirms that the FeSe is in the thin film limit.

To study the electronic property of the FeSe, we performed a differential conductance $(d I / d V)$ spectroscopy using a standard lock-in technique [30]. To maximize the energy resolution in measuring $d I / d V$ spectrum at our experiment temperature, we used a $\mathrm{Pb}$-coated superconducting tip [22,30]. Figure 1(e) shows the $d I / d V$ spectra measured in the FeSe and $\mathrm{Pb}$. For the FeSe spectrum, there is a characteristic peak near the bias voltage $\left(V_{\text {bias }}\right)$ of $-0.3 \mathrm{~V}$, which agrees with the spectra of FeSe in the literature $[35,39]$.

When the spectrum is enlarged around the Fermi energy, a superconducting gap is found [the inset of Fig. 1(e)]. Given by the following three facts, we conclude that the superconductivity of our FeSe is proximity induced from the $\mathrm{Pb}$ substrate. First, the superconducting coherence length $(\xi)$ of $\mathrm{Pb}(\sim 830 \AA)$ is much larger than the thickness of the FeSe ( $\sim 16 \AA$ for 3 TL). Second, the gap is fully developed revealing the $s$-wave nature in superconductivity. Third, the phonon peaks associated with $\mathrm{Pb}$ superconductivity are clearly seen in the spectrum of FeSe [marked by arrows in the inset of Fig. 1(e)] [40,41]. No hint of unconventional superconductivity is observed. The gap size $(4.6 \mathrm{meV})$ is twice that of the $\mathrm{Pb}$ superconducting gap $(2 \Delta \approx 2.3 \mathrm{meV})$ because the tip is coated with $\mathrm{Pb}$. Under the $T_{c}$ of $\mathrm{Pb}$ superconductivity $(\sim 7.2 \mathrm{~K})$, all electrons of FeSe are forced to participate in the proximity-induced $s$-wave pairing. Any electron pairs which are not in a time reversal symmetry (TRS) relationship will form YSR excitation states within the superconducting gap [21,22,42].

We have investigated the response of FeSe to proximityinduced $s$-wave superconductivity. Figure 2(b) shows spectra measured at different FeSe sites marked with $A$, $B, C$, and $R$ in Fig. 2(a). The spectrum on $\mathrm{PbSe}$ (position $R$ ) is first measured as a reference because PbSe is not an intrinsic superconductor and thus its superconductivity is undoubtedly induced by proximity to the $\mathrm{Pb}$. Remarkably, the spectrum measured inside the FeSe island (position $B$ ) exhibits no YSR excitation, indicating that the expected magnetic moment is zero for the ground state. This is microscopic evidence of the absence of static magnetic ordering in FeSe. In contrast, the spectra measured at the boundary of the FeSe island (positions $A$ and $C$ ) show strong YSR excitation states, suggesting local magnetic moments are developed along the boundary. The result demonstrates that $\mathrm{FeSe}$ is not a conventional nonmagnetic material although its ground state preserves TRS $[9,12,13,43-45]$. Figure 2(c) displays the $d I / d V$ plot along the dashed line in Fig. 2(a). We barely observed a variation in superconductivity inside the FeSe island. 

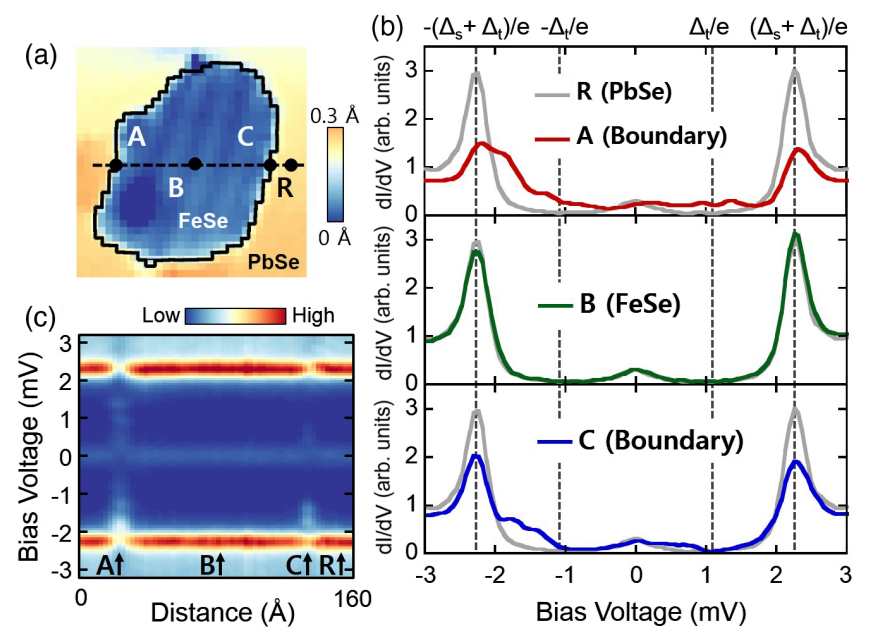

FIG. 2. (a) The FeSe grown on $\mathrm{Pb}(111) . V_{\text {bias }}=-0.1 \mathrm{~V}$ and $I=50 \mathrm{pA}$. (b) The $d I / d V$ spectra measured at points $A, B, C$, and $R$ marked in (a). $V_{\text {bias }}=-3.0 \mathrm{mV}$ and $I=50 \mathrm{pA}$. Lock-in: $V_{\text {rms }}=60 \mu \mathrm{V}$. Because the Pb-coated superconducting tip is used, the coherent peaks are located at $E= \pm\left(\Delta_{s}+\Delta_{t}\right) . \Delta_{s}$ and $\Delta_{t}$ are the superconducting gap of the sample and the tip, respectively. The broad peak around $V_{\text {bias }}=0 \mathrm{mV}$ is due to the thermal effect at $4.3 \mathrm{~K}$. (c) Line $d I / d V$ spectroscopy measured along the dashed line in (a).

To study the impurity-induced quantum instability in FeSe, we have deposited $\mathrm{Ag}$ atoms onto the sample at $20 \mathrm{~K}$ [Fig. 3(a)]. The height of Ag atoms is $\sim 0.7 \AA$ on the FeSe [Fig. 3(b)]. By careful Fourier transform analysis, we determined that the $\mathrm{Ag}$ atoms are located on the center of top Se lattice [Fig. 3(c) and Supplemental Material [26] ]. Nonmagnetic atoms, such as Ag, will not break TRS and thus the $s$-wave superconductivity should not respond to them. Accordingly, the $d I / d V$ spectrum of the Ag atom on the $\mathrm{Pb}$ surface exhibits no YSR excitation [Fig. 3(d)]. We only observed a slight variation in gap size, which might be related to the double Fermi surface of $\mathrm{Pb}$ but is not caused by magnetism [46]. By contrast, when we measured the $d I / d V$ spectrum on the Ag atom placed on the FeSe, strong YSR excitation is detected, showing that local magnetic moments are developed. We also observed similar YSR excitation for $\mathrm{Au}$ adatoms on the FeSe (Supplemental Material [26]). It is remarkable that such nonmagnetic atoms induce local magnetism in $\mathrm{FeSe}$, which supports the assertion that the ground state of FeSe is near a magnetic quantum critical point.

To understand the pattern of local magnetic moments, we measured $d I / d V$ maps at the various energies $\left(E=\mathrm{eV}_{\text {bias }}\right)$ of YSR states. Figure 3(e) shows the topography of a $\mathrm{Ag}$ atom and simultaneously obtained $d I / d V$ maps. The most striking feature in the $d I / d V$ maps is the $C_{2}$ symmetry. The $d I / d V$ patterns at $E=-1.85 \mathrm{meV}$ and $E=-1.3 \mathrm{meV}$ are split up and down. The $d I / d V$ pattern at $E=1.48 \mathrm{meV}$ is slightly tilted from the up-and-down splitting. These are representative magnetic patterns induced by $\mathrm{Ag}$ atoms in
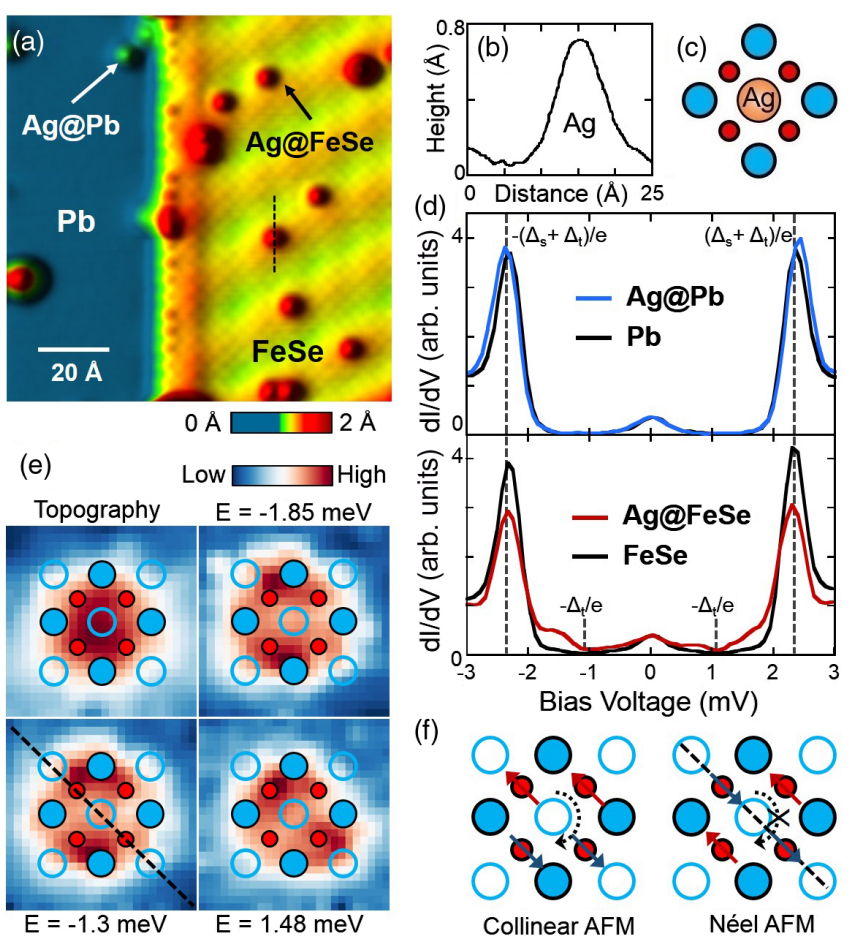

FIG. 3. (a) Topography of $\mathrm{Ag}$ atoms on the FeSe and $\mathrm{Pb}$ surface. The moiré pattern is seen in the FeSe along diagonal direction. $V_{\text {bias }}=-0.1 \mathrm{~V}$ and $I=50 \mathrm{pA}$. (b) The height profile along the dashed line in (a). (c) The Ag atom (orange ball) is placed on the center of top Se lattice. (d) The Ag atom on $\mathrm{Pb}$ do not show in-gap states excitation. By contrast, the Ag atoms on FeSe show strong in-gap states excitation. $V_{\text {bias }}=-3.0 \mathrm{mV}$ and $I=50$ pA. Lock-in: $V_{\text {rms }}=60 \mu \mathrm{V}$. (e) Topography of the $\mathrm{Ag}$ atom and $d I / d V$ maps (size: $9.5 \AA \times 9.5 \AA$ ). (f) Two spin models; collinear AFM model preserves the $C_{2}$ symmetry imposed by the lattice, as indicated by the dotted arrow. The Néel AFM model breaks the $C_{2}$ symmetry while it maintains mirror symmetry marked with the dashed line.

FeSe (Supplemental Material [26]). In terms of the reliability of the observed $C_{2}$ symmetry, the magnetic patterns varied slightly depending on the $\mathrm{Ag}$ atom, but the overall $C_{2}$ symmetry was maintained for the majority of Ag atoms in the repeated experiments (Supplemental Material [26]). We also exclude the moiré pattern as an origin of the splitting [26].

Several features are present regarding the magnetic patterns. First, the tendency of splitting is the same among the $\mathrm{Ag}$ atoms placed on the FeSe in Fig. 3(a). No Ag atom showed a splitting along the left and right. This symmetry breaking can be attributed to the nematic order in our FeSe. Second, the in-gap states are strongly localized near the Ag atom. We barely observed long-range magnetic ordering near the $\mathrm{Ag}$ atom. Third, the magnetic patterns do not follow the full symmetry of the crystal lattice but only satisfy $C_{2}$ symmetry.

Recent theory predicts that the local magnetic moments in FeSe reflect the momentum structure of the magnetic 
fluctuations in the bulk [19]. To confirm this, we compared the magnetic patterns with spin models for FeSe. Figure 3(f) shows the collinear AFM (CAFM) and Néel AFM models. The CAFM model preserves the $C_{2}$ symmetry of FeSe, which agrees with our magnetic patterns. The Néel AFM model can be ruled out because it does not have $C_{2}$ symmetry and it has a definite mirror symmetry plane [the dashed line in Fig. 3(f)] that contradicts the symmetry of the magnetic patterns at $E=-1.85 \mathrm{meV}$ and $E=-1.3 \mathrm{meV}$. Therefore, although we have not observed a long-range magnetic ordering near the impurities, the magnetic patterns do reflect the symmetry of the CAFM ordering. Note that the spin angle of $45^{\circ}$ in our models is supported by recent $\mu \mathrm{SR}$ measurement [47]. For the spin angle of $0^{\circ}$, however, the magnetic patterns are also consistent with the symmetry of the CAFM ordering (Supplemental Material [26]).

Now we discuss the origin of CAFM order when local magnetic moments are induced by impurities in FeSe. A recent theoretical study, based on a multiorbital Hubbard model with a band structure relevant for FeSe, mapped out the phase diagram of local impurity-induced magnetism [19]. Importantly, as shown in Ref. [19], the orbital selectivity characteristic of Hund's metals [33,48] is directly imprinted on the local impurity-induced order, yielding local $(\pi, 0)$ AFM structure versus $(\pi, \pi)$ AFM local order when orbital selectivity is included or disregarded, respectively. These calculations reveal that strongly anisotropic magnetic fluctuations dictate the detailed structure of induced local magnetic order [19]. A similar transmutation of the structure of the bulk magnetic fluctuations and superconducting pairing, takes place when including orbital selectivity $[29,45,49]$. While the results from Ref. [19] focused on impurities centered on Fe sites, we show in the Supplemental Material that a Se-centered disorder like $\mathrm{Ag}$ also induces local $(\pi, 0)$-structured magnetic order. In addition, we have applied the same theoretical machinery to sample edges, and found that FeSe is very susceptible to inducing magnetism strongly localized near the edges (Supplemental Material [26]), in agreement with our STM findings.

We occasionally found a dumbbell-shaped local defect in the FeSe before the deposition of Ag atoms [Fig. 4(a)]. The center of the defect is located at the Fe site as guided by the two dashed lines depicted in Fig. 4(a), suggesting it is an $\mathrm{Fe}$ vacancy $[32,50,51]$. When the $d I / d V$ spectrum is measured off the Fe defect, no YSR excitation is observed. However, when it is measured on the defect, strong YSR excitation is observed, indicating that an $\mathrm{Fe}$ defect also induces local magnetic moments in FeSe.

Figure 4(c) shows the topography of the Fe defect and simultaneously measured $d I / d V$ maps. The dashed line depicted represents the mirror symmetry plane imposed by the crystal lattice. The topography is naturally symmetric with respect to this mirror symmetry. Interestingly, the

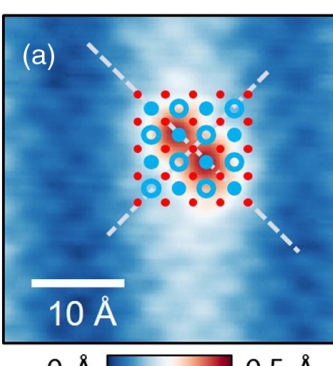

$0 \AA ⿻ 0.5 \AA$

(c)

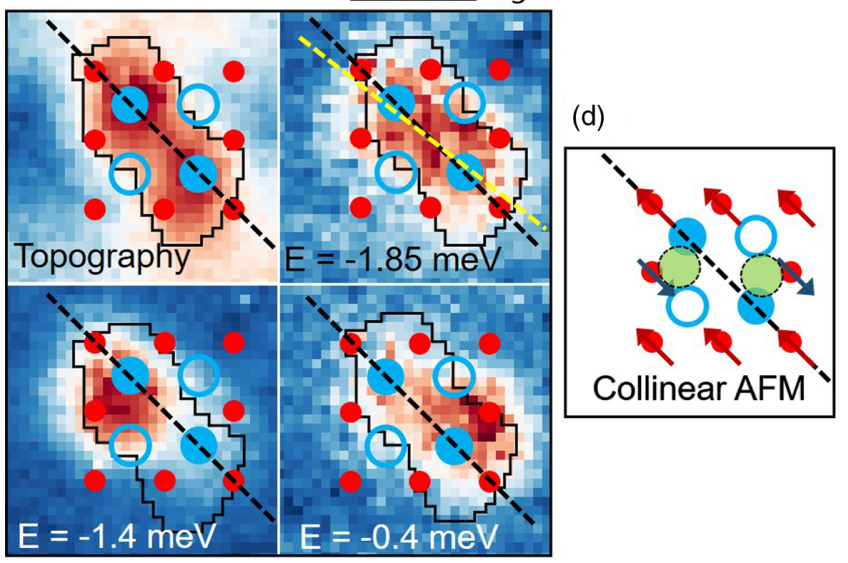

FIG. 4. (a) Topography of an Fe defect in the FeSe. $V_{\text {bias }}=$ $-30 \mathrm{mV}$ and $I=50 \mathrm{pA}$. (b) On the defect site, strong in-gap state excitation is observed. No in-gap states are present off the defect site. $V_{\text {bias }}=-3.0 \mathrm{mV}$ and $I=50 \mathrm{pA}$. Lock-in: $V_{\text {rms }}=60 \mu \mathrm{V}$. (c) Topography of the Fe defect and simultaneously obtained $d I / d V$ maps. The dashed line represents the mirror symmetry plane in topography. The yellow dashed line denotes the axis of the magnetic pattern at $E=-1.85 \mathrm{meV}$. (d) The collinear AFM model breaks the $C_{2}$ symmetry around the defect as well as the mirror symmetry.

magnetic patterns in the $d I / d V$ maps are not symmetric under the mirror operation $[17,48]$. Recent theory shows that orbital selectivity can give rise to chiral patterns in the conductance maps from local magnetic ordering near $\mathrm{Fe}$ defects, upon which the mirror symmetry is broken [19]. In fact, the $d I / d V$ map at $E=-1.85 \mathrm{meV}$ shows the axis of the magnetic pattern (yellow dotted line) is tilted from the mirror symmetry axis according to the theory. We find that the observed magnetic patterns are again consistent with the symmetry of the CAFM model. Figure 4(d) shows the CAFM model with the Fe defect. The CAFM ordering directly breaks the mirror symmetry of the crystal lattice. Furthermore, the CAFM ordering breaks the $C_{2}$ symmetry of FeSe when a defect exists in the Fe site, which is in contrast to the case of the $\mathrm{Ag}$ on FeSe. Two green-colored sites in Fig. 4(d) are then no longer equivalent in terms of symmetry. In the experiment, the magnetic excitation at these sites indeed appears at different energies as shown in Fig. 4(c) $(E=-1.4 \mathrm{meV}$ and $E=-0.4 \mathrm{meV})$.

Our STM experiment provides a novel method to study local magnetism in correlated superconductors, here 
exemplified through FeSe. The results lead to several important remarks. First, the magnetic quantum phase transition by nonmagnetic impurities is microscopically observed in FeSe. Second, we show that the magnetic patterns of the local magnetic moments are consistent with the $(\pi, 0)$ AFM phase, implying that the orbital selectivity is at play. Third, our experiment reveals the magnetic characteristics of impurities in FeSe. The sign-changing superconductivity responds to both magnetic and nonmagnetic impurities, whereas the $s_{++}$superconductivity only responds to magnetic impurities [21-25,52-56]. It is therefore important to characterize the magnetic property of impurities before they are used to probe the symmetry of superconductivity. In our experiment we have unambiguously observed signatures of magnetism on crystalline defects like the crystal boundary and Fe vacancy in $\mathrm{FeSe}$, a property which has not been considered earlier $[57,58]$. Furthermore, it should be noted that the local magnetism can also be induced by nonmagnetic impurities in FeSe.

The data analysis described here is based on a simple and powerful symmetry argument. A theoretical work deserving further investigation is identifying the $\mathrm{Fe}$ orbitals responsible for magnetic patterns observed in the experiment. This will reveal the origin of the local magnetism in FeSe in conjunction with the orbital selectivity. In future STM works, it will be interesting to study how the local magnetism develops into the bulk magnetism when the $\mathrm{Ag}$ impurities form networks, which can be accomplished by the STM atom manipulation. Nearby, it might be possible to detect strong orbital-selective spin fluctuations through inelastic tunneling spectroscopy, which could be in turn related to the anisotropic Cooper pairing in $\mathrm{FeSe}$ [59].

This work has been supported by Samsung Science \& Technology Foundation under Project No. SSTF-BA150204. The Center for Nanostructured Graphene (CNG) is supported by the Danish National Research Foundation, Project No. DNRF103. B. M. A. acknowledges support from the Independent Research Fund Denmark, Grant No. DFF-8021-00047B.

*jseo@dgist.ac.kr

[1] A. I. Coldea and M. D. Watson, Annu. Rev. Condens. Matter Phys. 9, 125 (2018).

[2] A. E. Böhmer and A. Kreisel, J. Phys. Condens. Matter 30, 023001 (2018).

[3] T. M. McQueen, A. J. Williams, P. W. Stephens, J. Tao, Y. Zhu, V. Ksenofontov, F. Casper, C. Felser, and R. J. Cava, Phys. Rev. Lett. 103, 057002 (2009).

[4] M. Bendele, A. Amato, K. Conder, M. Elender, H. Keller, H.-H. Klauss, H. Luetkens, E. Pomjakushina, A. Raselli, and R. Khasanov, Phys. Rev. Lett. 104, 087003 (2010).

[5] T. Imai, K. Ahilan, F. L. Ning, T. M. McQueen, and R. J. Cava, Phys. Rev. Lett. 102, 177005 (2009).
[6] J.-H. She, M. J. Lawler, and E.-A. Kim, Phys. Rev. Lett. 121, 237002 (2018).

[7] F. Wang, S. A. Kivelson, and D.-H. Lee, Nat. Phys. 11, 959 (2015).

[8] J. K. Glasbrenner, I. I. Mazin, H. O. Jeschke, P. J. Hirschfeld, R. M. Fernandes, and R. Valentí, Nat. Phys. 11, 953 (2015).

[9] R. M. Fernandes, A. V. Chubukov, and J. Schmalian, Nat. Phys. 10, 97 (2014).

[10] S. H. Baek, D. V. Efremov, J. M. Ok, J. S. Kim, J. van den Brink, and B. Büchner, Nat. Mater. 14, 210 (2015).

[11] M. Bendele, A. Ichsanow, Yu. Pashkevich, L. Keller, Th. Strässle, A. Gusev, E. Pomjakushina, K. Conder, R. Khasanov, and H. Keller, Phys. Rev. B 85, 064517 (2012).

[12] J. P. Sun et al., Nat. Commun. 7, 12146 (2016).

[13] K. Kothapalli et al., Nat. Commun. 7, 12728 (2016).

[14] T. Terashima et al., J Phys. Soc. Jpn. 84, 063701 (2015).

[15] R. Khasanov et al., Phys. Rev. B 78, 220510(R) (2008).

[16] M. He, L. Wang, F. Hardy, L. Xu, T. Wolf, P. Adelmann, and C. Meingast, Phys. Rev. B 97, 104107 (2018).

[17] W. Li et al., Nat. Phys. 13, 957 (2017).

[18] M. N. Gastiasoro and B. M. Andersen, J. Supercond. Novel Magn. 28, 1321 (2015).

[19] J. H. J. Martiny, A. Kreisel, and B. M. Andersen, Phys. Rev. B 99, 014509 (2019).

[20] M. N. Gastiasoro, P. J. Hirschfeld, and B. M. Andersen, Phys. Rev. B 88, 220509(R) (2013).

[21] A. Yazdani, B. A. Jones, C. P. Lutz, M. F. Crommie, and D. M. Eigler, Science 275, 1767 (1997).

[22] K. J. Franke, G. Schulze, and J. I. Pascual, Science 332, 940 (2011).

[23] H. Shiba, Prog. Theor. Phys. 40, 435 (1968).

[24] M. I. Salkola, A. V. Balatsky, and J. R. Schrieffer, Phys. Rev. B 55, 12648 (1997).

[25] A. V. Balatsky, I. Vekhter, and J.-X. Zhu, Rev. Mod. Phys. 78, 373 (2006).

[26] See Supplemental Material at http://link.aps.org/ supplemental/10.1103/PhysRevLett.124.117001 for details, which includes Refs. [19,27-29].

[27] A. Weiße, G. Wellein, A. Alvermann, and H. Fehske, Rev. Mod. Phys. 78, 275 (2006).

[28] L. Covaci, F. M. Peeters, and M. Berciu, Phys. Rev. Lett. 105, 167006 (2010).

[29] A. Kreisel, B. M. Andersen, P. O. Sprau, A. Kostin, J. C. Séamus Davis, and P. J. Hirschfeld, Phys. Rev. B 95, 174504 (2017).

[30] S. Y. Song and J. Seo, Sci. Rep. 7, 12177 (2017).

[31] C.-L. Song et al., Science 332, 1410 (2011).

[32] P. Choubey, T. Berlijn, A. Kreisel, C. Cao, and P. J. Hirschfeld, Phys. Rev. B 90, 134520 (2014).

[33] A. Kostin, P. O. Sprau, A. Kreisel, Y.X. Chong, A. E. Böhmer, P. C. Canfield, P. J. Hirschfeld, B. M. Andersen, and J. C. S. Davis, Nat. Mater. 17, 869 (2018).

[34] F. S. Li et al., 2D Mater. 3, 024002 (2016).

[35] X. Liu, L. Zhao, S. He, J. He, D. Liu, D. Mou, B. Shen, Y. Hu, J. Huang, and X. J. Zhou, J. Phys. Condens. Matter 27, 183201 (2015).

[36] A. Cavallin, V. Sevriuk, K. N. Fischer, S. Manna, S. Ouazi, M. Ellguth, C. Tusche, H. L. Meyerheim, D. Sander, and J. Kirschner, Surf. Sci. 646, 72 (2016). 
[37] A. Eich et al., Phys. Rev. B 94, 125437 (2016).

[38] U. R. Singh, J. Warmuth, V. Markmann, J. Wiebe, and R. Wiesendanger, J. Phys. Condens. Matter 29, 025004 (2017).

[39] Y. H. Yuan et al., Nano Lett. 18, 7176 (2018).

[40] M. Schackert, T. Märkl, J. Jandke, M. Hölzer, S. Ostanin, E. K. U. Gross, A. Ernst, and W. Wulfhekel, Phys. Rev. Lett. 114, 047002 (2015).

[41] W. L. McMillan and J. M. Rowell, Phys. Rev. Lett. 14, 108 (1965).

[42] D. J. Choi, C. Rubio-Verdu, J. de Bruijckere, M. M. Ugeda, N. Lorente, and J. I. Pascual, Nat. Commun. 8, 15175 (2017).

[43] Q. Wang et al., Nat. Commun. 7, 12182 (2016).

[44] Q. Wang et al., Nat. Mater. 15, 159 (2016).

[45] T. Chen et al., Nat. Mater. 18, 709 (2019).

[46] M. Ruby, B. W. Heinrich, J. I. Pascual, and K. J. Franke, Phys. Rev. Lett. 114, 157001 (2015).

[47] R. Khasanov, Z. Guguchia, A. Amato, E. Morenzoni, X. Dong, F. Zhou, and Z. Zhao, Phys. Rev. B 95, 180504(R) (2017).

[48] P. O. Sprau, A. Kostin, A. Kreisel, A. E. Böhmer, V. Taufour, P. C. Canfield, S. Mukherjee, P. J. Hirschfeld,
B. M. Andersen, and J.C. Séamus Davis, Science 357, 75 (2017).

[49] A. Kreisel, B. M. Andersen, and P. J. Hirschfeld, Phys. Rev. B 98, 214518 (2018).

[50] S. Chi et al., Phys. Rev. B 94, 134515 (2016).

[51] D. Huang, T. A. Webb, C.-L. Song, C.-Z. Chang, J. S. Moodera, E. Kaxiras, and J. E. Hoffman, Nano Lett. 16, 4224 (2016).

[52] S. H. Pan, E. W. Hudson, K. M. Lang, H. Eisaki, S. Uchida, and J. C. Davis, Nature (London) 403, 746 (2000).

[53] H. Yang, Z. Wang, D. Fang, Q. Deng, Q.-H. Wang, Y.-Y. Xiang, Y. Yang, and H.-H. Wen, Nat. Commun. 4, 2749 (2013).

[54] Y. J. Yan et al., Phys. Rev. B 94, 134502 (2016).

[55] Z. Du et al., Nat. Phys. 14, 134 (2017).

[56] C. Liu, Z. Wang, Y. Gao, X. Liu, Y. Liu, Q.-H. Wang, and J. Wang, Phys. Rev. Lett. 123, 036801 (2019).

[57] L. Jiao, S. Rößler, C. Koz, U. Schwarz, D. Kasinathan, U. K. Rößler, and S. Wirth, Phys. Rev. B 96, 094504 (2017).

[58] Z. Ge, C. Yan, H. Zhang, D. Agterberg, M. Weinert, and L. Li, Nano Lett. 19, 2497 (2019).

[59] S. Chi et al., Nat. Commun. 8, 15996 (2017). 